\title{
Challenges and opportunities in solid-state chemistry ${ }^{\star}$
}

\author{
Francis J. DiSalvo ${ }^{\dagger}$ \\ Department of Chemistry and Chemical Biology, Cornell University, Ithaca, NY \\ 14853 USA
}

\begin{abstract}
We are largely unable to predict the composition and crystal structure of new extended structure compounds. Yet we find that as materials become more complex in stoichiometry and structure, new, interesting, and perhaps useful, phenomena are encountered. The need for improved experimental and theoretical approaches to seize more rapidly the opportunities provided in this complexity is discussed.
\end{abstract}

\section{INTRODUCTION}

We increasingly live in a materials world, yet we remain largely ignorant of the chemical possibilities inherent in the periodic table of elements. Here we discuss one branch of chemistry, the chemistry of nonmolecular solids (so-called extended structure solids). We show that predicting even basic features such as composition and crystal structure of new solids is largely impossible. Since composition and structure are required inputs for the prediction of properties, we usually discover materials with enhanced and especially with novel properties by chance rather than design. We are still practicing a very Edisonian or empirical science, especially in the synthesis of novel phases, which is largely "accidental". While many exciting discoveries have come from this approach, one hopes for a more rational strategy. Such a strategy needs to be developed if we are to make significant progress in exploring the huge number of possibilities inherent in the periodic table.

Scientists have now discovered the composition and often the structure of about 20 million different substances. More than $95 \%$ of these are molecular organic compounds, based on the chemistry of carbon and a few other elements such as hydrogen, oxygen, nitrogen, and sulfur. Many of these are known to result from biological processes in plants or animals, but an increasing number are new molecules synthesized only in the laboratory. A much smaller number of compounds contain one or more of the metallic elements. Yet, these elements are the majority of those in the periodic table. Again, a large fraction of these metal-containing compounds, inorganic compounds, are molecular in structure. Only a very small fraction of known compounds, perhaps $1 \%$ of the total, are inorganic and exhibit nonmolecular structures. In such compounds the chemical bonds extend from one atom to the next across the macroscopic dimensions of the material. Such compounds include familiar materials, such as diamond, iron oxide, and table salt. In spite of their relatively small number, these inorganic extended structure materials are the basis of many technologies essential to our economy and well-being. I only need to mention the broad impact of several classes of materials, such as ceramics, metals and their alloys, and semiconductors, to make the point. Of course, organic materials, especially polymers, are also exploited in many technologies. But we leave the discussion of such carbon-based materials to another forum.

\footnotetext{
*Plenary lecture presented at the $16^{\text {th }}$ IUPAC Conference on Chemical Thermodynamics (ICCT-2000), Halifax, Nova Scotia, Canada, 6-11 August 2000. Other presentations are published in this issue, pp. 1799-2082.

†E-mail: fjd3@cornell.edu
} 
Focusing on extended structure solids, we can further divide these compounds into two broad groups: valence compounds and intermetallic compounds. Figure 1 shows the electronegativity of each element in the periodic table, the higher the value of the electronegativity, the larger the font used. Valence compounds are formed by combining electropositive elements (elements near or to the left of the metalloid line, the bold zig-zag line in Fig. 1) with electronegative elements to the right of the metalloid line. Such solids include $\mathrm{NaCl}, \mathrm{NbN}$, and $\mathrm{SiO}_{2}$, for example. Intermetallic solids are formed from elements near the metalloid line and to the left; such as: $\mathrm{CsAu}, \mathrm{NiZr}, \mathrm{CrB}_{2}$, and $\mathrm{V}_{3} \mathrm{Si}$.

\section{"SIMPLE" CHALLENGES: COMPOSITION AND STRUCTURE}

Generally, oxidation states are useful and easy to assign in valence compounds, especially in compounds between metals and the most electronegative elements ( $, ~ O, F, S, C l, S e, B r$ and I). The composition of such compounds is usually consistent with integer oxidation states, which can be simply determined from the number of valence electrons. The composition of binary valence compounds can thus be "predicted" based on oxidation states. Of course, we already have prepared essentially all such binary compounds, so we already know the answer. But for ternary valence compounds, oxidation state rules often do not help. As an example, consider the ternary compounds that can be prepared by reaction of $\mathrm{Li}_{2} \mathrm{O}$ and $\mathrm{MoO}_{3}$. In this case, we fix the oxygen content so that the oxidation states of $\mathrm{Li}(+1)$ and of Mo (+6) remain fixed. There are 3 known compounds [1]: $\mathrm{Li}_{2} \mathrm{MoO}_{4}\left(\mathrm{Li}_{2} \mathrm{O}: \mathrm{MoO}_{3}:: 1: 1\right)$, $\mathrm{Li}_{2} \mathrm{Mo}_{4} \mathrm{O}_{13}(1: 4)$, and $\mathrm{Li}_{4} \mathrm{Mo}_{5} \mathrm{O}_{17}$ (2:5). If one combines these reactants at other ratios, mixtures of the ternaries and/or unreacted excess reactants result. How does nature choose these ratios for the ternaries? We can say that these ratios represent the minimum in the free energy at that composition, but this is not predictive. On the other hand, if we know the composition and structure of the binaries and ternaries, we can calculate with some confidence the phase diagram. But this is not useful when we want to predict possible new compounds, especially those with previously unknown crystal structures.

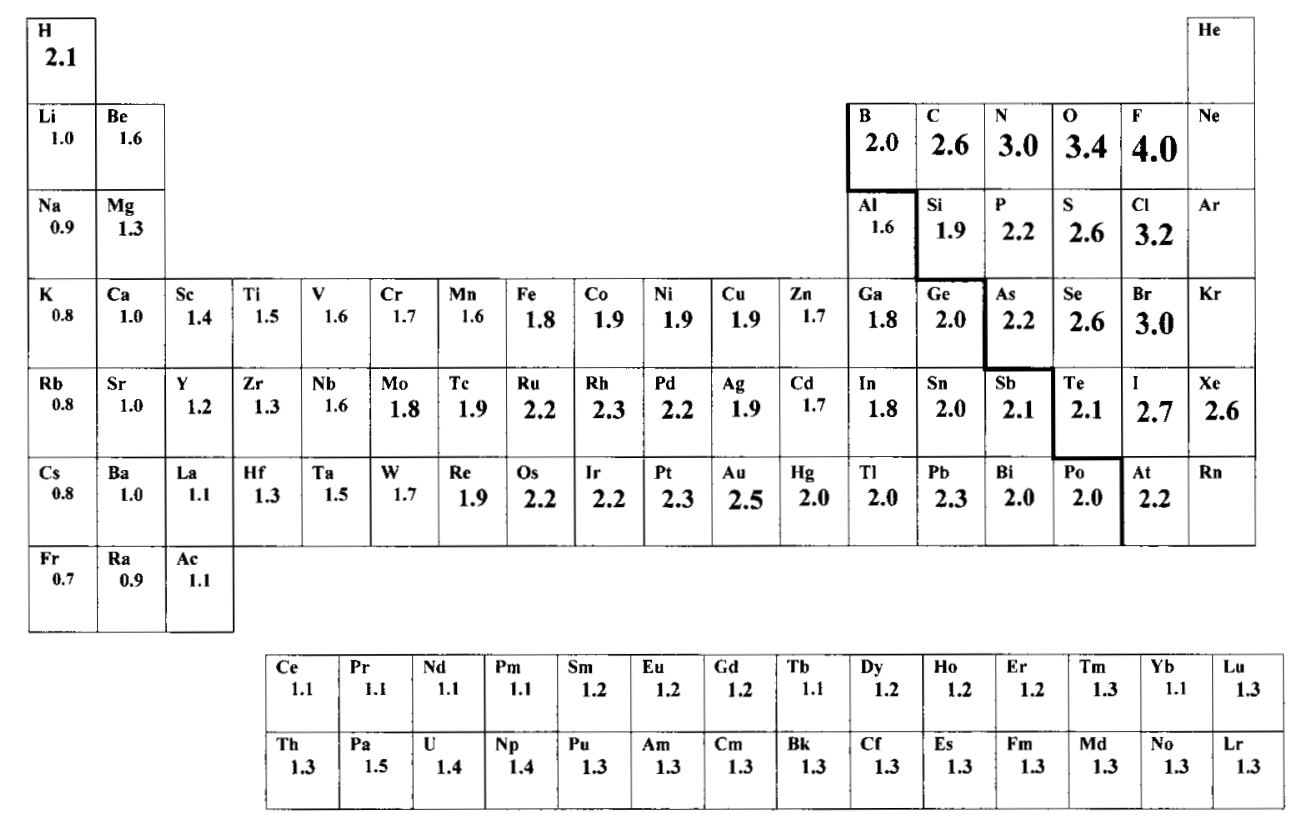

Fig. 1 The electronegativity of each element in the periodic table is shown in a point size proportional to the value of electronegativity. Except for the precious metals, metals generally have an electronegativity less than 2.0 and are termed "electropositive", while those with electronegativities greater than 2.5 are to the right of the bold line (metalloid line) and are termed electronegative. The metalloid elements are those near the metalloid line; their chemical behavior varies greatly, depending on which elements they are combined with. 
The situation is even more difficult with intermetallic compounds, since oxidation state rules cannot be usefully applied when the electronegativity difference is small. Consider the products of reacting $\mathrm{Ni}$ and $\mathrm{Zr}$ at different ratios: $\mathrm{Ni}_{5} \mathrm{Zr}, \mathrm{Ni}_{7} \mathrm{Zr}_{2}, \mathrm{Ni}_{3} \mathrm{Zr}, \mathrm{Ni}_{21} \mathrm{Zr}_{8}, \mathrm{Ni}_{10} \mathrm{Zr}_{7}, \mathrm{Ni}_{11} \mathrm{Zr}_{9}, \mathrm{NiZr}$, and $\mathrm{NiZr}_{2}[2,3]$. What strange and wonderful compositions! Each has its own ordered arrangement of atoms. Several of them exist over a range of composition: $\mathrm{Ni}_{1-x} \mathrm{Zr}_{x}$, where $x$ can vary by a few atomic percent, while others allow very little variation in composition. But again we have no a priori rules to predict these compositions or their structures. So let us consider next what we do know from our past synthetic research.

Of course, the composition of elemental materials is not an issue. The structures are largely simple-often derived from close packing — and often high symmetry (such as face-centered cubic, bodycentered cubic, or hexagonal). There may be a few surprises left, for example, the existence of metastable substances such as bucky-balls $\left(\mathrm{C}_{60}\right)$, but we essentially know the answer here and often teach some of these common structures to beginning college chemistry students.

Binary compounds have also been largely explored. We can simply calculate the number of paired combinations that we needed to study. For this purpose we use a truncated periodic table: we discard unreactive elements such as $\mathrm{He}$ and $\mathrm{Ne}$ and we discard radioactive elements because they are investigated in only a few places and because they have little commercial use. Such a periodic table contains 80 elements, plus or minus a few. So, for binary compounds we have 80 elements to choose from for the first and 79 for the second element. This gives $80 \times 79 / 2=3160$ combinations (divide by 2 , since the order in which we choose the elements is irrelevant). Most of these have been examined in some detail, and we know what equilibrium phases are present in the phase diagrams [2,3]. [Phase diagrams show more than the composition of the different binary compounds. They also show the range of thermal stability (such as melting or decomposition points) and other thermodynamically important events as a function of composition, temperature, and/or pressure.] Villars and co-workers have analyzed this group of compounds (on the order of 30000 compounds) and report some interesting observations [4-7]. First, about $90 \%$ of binary compounds have simple stoichiometries: $\mathrm{MX}, \mathrm{MX}_{2}, \mathrm{MX}_{3}$, or $\mathrm{M}_{3} \mathrm{X}_{5}$ ( $\mathrm{M}$ and $\mathrm{X}$ stand for the two elements in the compound). There are strange stoichiometries, as illustrated above in the $\mathrm{Ni}-\mathrm{Zr}$ system, but these are less common.

However, the number of structure types adopted by the common binary compositions is small, only about 80 types. For example, for compounds with the MX composition there are 20 common structure types. That is, many different compounds crystallize in the same structural arrangement. If you know the $\mathrm{NaCl}$ structure, you would not be surprised to learn that $\mathrm{KCl}$ has the same structure. $\mathrm{But} \mathrm{BaO}$, $\mathrm{LaN}, \mathrm{LaSb}, \mathrm{NbC}, \mathrm{ReC}$, and over 200 other compounds also adopt the same structure [3], which is called the Sodium Chloride Structure Type. (Note that this structure type is adopted by compounds with quite different bonding interactions from ionic to covalent/metallic.) This small number of structure types greatly simplifies the study of binary phases. Many researchers have constructed empirical "rules" that "explain" the adoption of particular structures as a function of a few variables. These include the early works of Hume-Rothery [8], Mooser and Pearson [9], Miedema [10], and Brewer [11]. More recently, using an extensive database, Villars and collaborators also found that the adoption of a particular structure type could be "predicted" (with about 95\% confidence, but of course, retrospectively). This prediction is based on only three atomic parameters that characterize the two elements: the total valence electron count, the electronegativity difference and the difference, in radii of the valence orbitals.

These approaches are largely empirical. While they are also largely retrospective, especially the Villars method may have some utility in making a good guess of possible structures in the few remaining binary phase diagrams that are incomplete. One could take a more basic approach and try to use quantum mechanics to predict the composition and structure of new compounds. This challenge is presently way beyond even the most advanced computers and algorithms. Perhaps in the next decade or two enough progress will be made that such theoretical approaches will be helpful, but that is not certain. However, present methods are capable of addressing simpler questions; such as, "if the composition is known, which of the presently known structures adopted at that composition have the lowest energy?" Of course, if a new structure type is preferred, the calculation will not tell you that. Such meth-

(C) 2000 IUPAC, Pure and Applied Chemistry 72, 1799-1807 
ods are useful in special cases. For example, the structure of many binary solids will change when pressure is applied, often from one known type to another. The calculations that are presently possible are often able to predict the pressure(s) of transformation and the structures that are obtained [12]. But can this empirical or theoretical knowledge be used in predicting ternary phases?

\section{THE CURRENT FRONTIER: TERNARY COMPOUNDS}

There are many more ternary combinations of elements to consider: $80 \times 79 \times 78 / 6=82160$. In each of the 82169 cases we must construct the ternary phase diagram, an undertaking that is much larger than determining the binary diagram. Since less than $10 \%$ of these have been explored even partially [13], this may be considered the frontier of solid-state chemistry. That is not to say that quaternary, quinary, and higher compounds are unknown, but that even less is known about the possibilities inherent in mixing 4, 5, or more elements than in the case of ternaries. From the known ternaries (about 30000 of these are already known, even though most ternary systems have not been studied) we already see problems in trying to extrapolate from the knowledge of binaries. First, there are now about 1000 different ternary structure types known, and the list is growing fast. I emphasize that these are true ternary structures, in that the full structure is not observed when only two elements are present. In some cases, there are many representatives of some structure types, such as the approximately $500 \mathrm{com}$ pounds that adopt the $\mathrm{ThCr}_{2} \mathrm{Si}_{2}$ structure type, but in many other cases there are only a few known representatives of the structure type. Second, empirical correlations of structure type with atomic parameters are difficult to apply, since there are at least seven possible parameters based on valence electron count and pairwise electronegativity and radii differences. Furthermore, the utility of such empirical correlations generally relies on a database that is not too sparse. Since we are still discovering many new ternary structure types, we suspect that the database is indeed too sparse for such predictions in any case.

I suppose now is a good time to ask: if there are already so many known solid-state materials, why do we need more? Why do we need more compositionally and structurally complicated materials? Can't we just modify known materials by manipulating them on the nanoscale or by other suitable processing to obtain the desired behavior? Of course, the scientists' answer is sufficient for other scientists: because we want to know what is there. This answer, however, is generally insufficient for the general public, who fund our R\&D efforts. So, a more credible answer is: as materials become more complex in composition and especially in structure, there is evidence, at least in some cases, that enhanced or even novel properties result. Consider the copper oxide superconductors. The highest $T_{c}$ attainable is on the order of $40 \mathrm{~K}$ when three elements are present $\left(\mathrm{La}_{2} \mathrm{CuO}_{4+\mathrm{x}}\right)$, over $90 \mathrm{~K}$ when 4 elements are present $\left(\mathrm{YBa}_{2} \mathrm{Cu}_{3} \mathrm{O}_{7}\right)$, near $130 \mathrm{~K}$ when 5 elements are present $\left(\mathrm{Tl}_{2} \mathrm{Ba}_{2} \mathrm{Ca}_{2} \mathrm{Cu}_{3} \mathrm{O}_{7}\right)$, or near $165 \mathrm{~K}$ under pressure in $\mathrm{HgBa}_{2} \mathrm{Ca}_{2} \mathrm{Cu}_{3} \mathrm{O}_{\mathrm{x}}$ [14]. Each of these compounds adopts a structure type only seen (so far anyway!) when 3,4 , or 5 elements are present, respectively. Why should $\mathrm{T}_{\mathrm{c}}$ depend on this complexity? It is likely that the structural and compositional degrees of freedom that are allowed in more complicated structures allow the "tuning" of electronic factors to enhance the interactions responsible for the high $\mathrm{T}_{\mathrm{c}}$. So far as we know, this behavior cannot be (or at least has not been) obtained by changing the sample dimensions (e.g., going to the nanoscale) or by processing variables (introducing point or extended defects, etc.). While it is clear that some properties and phenomena depend critically on scale and processing, others depend on the complexity of the compound. But it is not at all clear what fraction of complex structures will support enhanced or novel phenomena rather than be uninteresting "bricks". Nor are we able to predict or choose which will be the interesting ones.

\section{BEYOND TERNARIES}

Let's assume we want more compositionally and structurally complex compounds. How many different combinations of elements do we need to explore as we go beyond the ternary case? Table 1 shows 
the result of the combinatorial calculations. It is easy to see that the number of combinations to study becomes very large, very quickly. If, for example, we make the foolish extrapolation of $\mathrm{T}_{\mathrm{c}}$ for the copper oxides vs. the number of elements essential to make the structure, we might conclude that it will take a compound with 8 elements to have a $T_{c}$ at room temperature. There are a huge number of combinations to consider-some 29 billion. Even if we restrict ourselves to compounds that contain 8 elements but 2 are copper and oxygen, there are still 300 million phase diagrams to examine for what may be a sparse phenomenon! Given the progress we have made so far in just exploring binaries and some ternaries, at the present rate it will take geologic times to sort through all these possibilities. Clearly, we must consider new ways to approach such problems.

Before we consider some possible approaches to this grand challenge, let us ask one more question. Just how complicated might the structures of compounds become? The driving force to produce multinary phases generally becomes smaller as the number of elements increases. So, for example, will we find that when we try to make compounds containing 5 essential elements that we most often make a mixture containing a binary and a ternary or several ternary compounds? To make a unique structure that requires, for example, $\mathrm{N}$ different elements, we can generally assume that each element will occupy a distinct crystallographic site (position). Compounds in which certain elements are randomly mixed on a given site (alloying or doping) usually adopt structures that are typical of compounds with $\mathrm{N}-1$ or $\mathrm{N}-2$, etc. elements. Elements prefer to occupy different sites when those elements have sufficiently different atomic properties, such as size, electronegativity, or number of valence electrons.

All 80 of the elements in our restricted periodic table are not sufficiently different that they will all occupy different sites. So again, just how complicated can we hope to get? Perhaps one hint comes from minerals. Here, nature has had some time to synthesize compounds from those elements available in the crust, as restricted by their concentration and distribution. Table 2 lists what I was able to glean from a crystallographic database [15]. First are two compounds that have the most cations on distinct crystallographic sites in a pure oxide. I could not find one that contained 7 distinct cations. So perhaps 6 cations is near the real limit in valence compounds. If we allow different anions (including polyatomic anions), then more complicated compounds are known, 6 of which are shown in Table 2. The limit here seems to be about 8 or 9 elements or a total of 10 simple cations and simple or polyatomic anions. Will we be able to beat nature because we can vary the concentrations of all the components and the preparation conditions over a larger range than is possible in nature? It may be some time before we have the experimental answer to that question. In any case, it seems very unlikely that unique structures will be adopted when we combine 20 or more elements, so the truly huge possibilities with 40 elements-

Table 1. Combinations of 80 elements.

\begin{tabular}{ll}
\hline Number of Elements & Combinations \\
\hline 2 & 3,160 \\
3 & 82,160 \\
4 & $1.58 \times 10^{6}$ \\
5 & $2.40 \times 10^{7}$ \\
6 & $3.00 \times 10^{8}$ \\
7 & $3.18 \times 10^{9}$ \\
8 & $2.90 \times 10^{10}$ \\
9 & $2.32 \times 10^{11}$ \\
10 & $1.65 \times 10^{12}$ \\
15 & $6.64 \times 10^{15}$ \\
20 & $3.45 \times 10^{18}$ \\
30 & $8.87 \times 10^{21}$ \\
40 & $1.07 \times 10^{23}$ \\
\hline
\end{tabular}


Table 2.

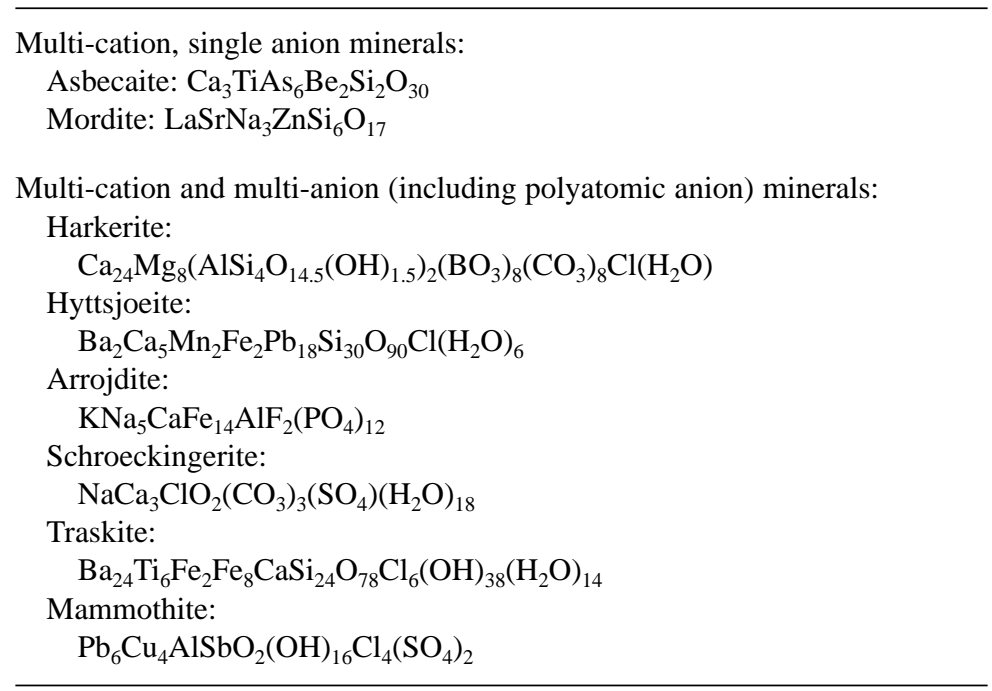

approaching Avogadro's number-seem very unlikely to be realized. Yet I believe (as a matter of faith!) that the possible combinations of up to 8 or 10 elements include compounds that are room-temperature superconductors.

\section{UNDERSTANDING THE PERIODIC TABLE AND INCREASING THE RATE OF DISCOVERY}

Now back to the challenge. Given the huge number of possibilities and the relatively small number of systems so far investigated, how can we improve our rate of discovery? Most likely, we have to learn to be much smarter about what we choose to prepare and at the same time much faster in preparation and examination of new materials. Can we do this? Being much smarter is a bigger challenge than doing things faster. Solid-state chemists have relied on experience and intuition to make progress to date. And this has worked reasonably well, not surprisingly, in areas where we collectively have the most experience, namely in oxide materials. Here, there are reasonably good sets of empirical rules and even some computational guides (as in the field of zeolite chemistry). There are, however, many phenomena in oxides that are poorly or incompletely understood, such as high-temperature superconductivity, metalinsulator transitions and high ionic conductivity. We still do not know how to design better oxide materials (or, for that matter, any materials) that will improve the properties in any of these areas. We need to make a more organized effort to push computational methods in the hopes that some predictive tools can be developed. To even hope to succeed, we will probably need to involve at least mathematicians, physicists, and chemists. The main problem is finding the absolute minimum free energy by varying the positions of a collection of different atoms. While some steps have been made in this direction [16], we are a long way from having a useful tool. This "global minimization problem" is not unique to materials chemistry, but also to many other important fields, such as the structure of proteins. Perhaps, if the problem is solved in one field, the solution can be applied to most of the others.

In the meanwhile, we can increase the speed at which we are exploring the periodic table. Even if predictive computational tools are developed, we will need to explore variations very rapidly to optimize properties. Further, computations themselves are unlikely to predict new phenomena. We will still need chemical insights to guide our thinking, because the combinations quickly become so large that at almost any speed we cannot and probably should not try to do everything. Nonetheless, while almost 
everyone can see the need to speed up the process, not everyone is sure how to do so (especially in the academic environment). There are different ways to approach this challenge. The approach depends on the objective and the available experimental "tools".

At one extreme we may want to understand the scientific issues in designing more complex structures. I might call this the academic approach. Then we clearly need not only to make materials at a faster rate, but we need to determine structures at a faster rate. With the use of the very intense X-ray beams at synchrotrons and of fast workstations, one could envision a center where one diffraction pattern could be collected and solved in a matter of a minute or less-perhaps in a few decades in a second or less! Of course, the tools to synthesize interesting samples at the same rate would need to be available as well. In fact, in many laboratories the current rate of synthesis of new compounds is slower than the rate of structure determination, due to advances in charge-couple device (CCD) detectors and computer control and analysis. And we would need to store, communicate, and digest all this information. But, little would be gained if this effort just provided a catalog of compounds and structures. Properties of at least some of these would need to be calculated and/or measured at a much faster rate than presently possible. If we do not measure all of them, how do we choose which to study? Grand challenges indeed.

The other extreme might be called the industrial or Edisonian approach. One does not initially care about structural or other details, but is guided by some property that is of interest and that can be rapidly measured. When an interesting or enhanced property is detected, one then may take the time to understand the underlying science of that case: the composition and structure and how that produces the property of interest. This information can then be used to rapidly screen for variations on the same theme.

Of course, some approach that is between these two could be designed. Indeed, a few commercial companies, such as Symyx Technologies in Santa Clara, California, are now trying to implement technologies to speed up the search (their approach is close to the industrial approach discussed above). Such an approach is now most commonly referred to as "combinatorial chemistry".

It is not clear how the traditional academic research group of a professor, a few graduate students and postdocs can implement the technology for combinatorial chemistry. However, in a few special cases, some academic laboratories have developed some tools that allow a combinatorial approach, for example, see refs. 17 and 18. There should be opportunities, however, for academic chemists to interact with industrial companies or government laboratories in productive ways for all to better exploit and develop combinatorial approaches.

Even with these approaches the possibilities remain almost endless. It would be pure hubris to assume that all the interesting or technologically useful materials and phenomena have been discovered. It is much more likely that we have barely scratched the surface of what is possible. In this short presentation, I hope to have excited you with that opportunity, not overwhelmed you with the challenges.

\section{POSTSCRIPTS}

Of course, there are many important issues left untouched in the above presentation. What about glasses and metastable crystalline materials? Such materials are prepared by controlling the kinetics of reaction. This is related to organic chemistry, where products are determined primarily by kinetic control of the interaction of different functional groups. There would be few organic compounds, if only those compounds were prepared that had the lowest free energy at that composition. So far, solid-state chemistry is largely concerned with the chemistry of thermodynamic products. Well, I am slighting the large science of glass, especially of silicate glasses. Glass science is technologically very important, but pursued in only relatively few academic laboratories. Will there be a large science of metastable crystalline solids? This will add yet another layer of complexity to the above challenges.

In many materials properties the microstructure is as important (or more important) as the composition or structure. So what about point defects, dislocations, and grain boundaries? Since these are 
affected by processing, how do we explore the role of processing? What about intentional structuring or compositional variations, as, for example, in quantum well devices? Will nanoscale structures be as interesting for these new materials as it appears to be for the relatively few materials systems studied so far? These are just a few of the untouched issues that come immediately to mind.

Also, those of us who are educators should take the time to explain to young students that we do not understand how the elements of the periodic table combine. We understand a lot about molecular species based on carbon, and we have a few other insights in a few restricted cases. But we teach beginning students as if all they need to know is this stuff that is perhaps 100 years old-and then they will be a chemist. Boring stuff-where is the challenge, the future? It is a wonder that any of them survive it! If we confessed our vast ignorance and pointed out the challenges and opportunities right from the beginning, perhaps we would snag more of the best and the brightest. They are probably the ones who will solve this great puzzle embodied in the periodic table. Indeed, I believe that this is one of the few grand challenges left in science.

\section{ACKNOWLEDGMENT}

Many colleagues have constructively commented on this manuscript. I thank them for their insights, but any mistakes or confusion are clearly my own. I especially mention Peter Battle, Simon Clarke, John Corbett, Miguel Alario Franco, Martha Greenblatt, Arthur Mar, Donald Murphy, and Mike O'Keeffe. I'm sure to have forgotten others, please forgive my absent-mindedness.

\section{REFERENCES AND NOTES}

1. See, for example: Phase Diagrams for Ceramists, Vol. III, p. 88, The American Ceramic Society.

2. See, for example: The Handbook of Binary Phase Diagrams, Genium Publishing Co.

3. For crystallographic information on intermetallic compounds and normal valence compounds (excluding oxides and halides), see: Pearson's Handbook of Crystallographic Data for Intermetallic Phases, Vols. 1-4, P. Villars and L. D. Calvert (Eds.), ASM International (1991).

4. A review of empirical methods can be found in MRS Bulletin, Vol 18, No. 2, February 1993, Materials Research Society.

5. P. Villars. J. Less-Common Met. 92, 215-238 (1983).

6. P. Villars. J. Less-Common Met. 99, 33-43 (1984).

7. P. Villars. J. Less-Common Met. 102, 199-211 (1984).

8. See, for example: The Structure of Metals and Alloys, 4th ed., Institute of Metals, London (1962).

9. E. Mooser and W. B. Pearson. Acta Crystallogr. 12, 1015 (1959).

10. A. R. Miedema. J. Less-Common Met. 46, 67 (1976).

11. L. Brewer. In Battelle Geneva Colloquium on Phase Stability in Metals and Alloys, P. Rudman, J. Stringer, R. L. Jaffee (Eds.), pp. 39-61, McGraw-Hill, NY (1967); see also L. Brewer. J. Am. Chem. Soc. 94, 1196 (1990).

12. P. Villars, A. Prince, H. Okamoto. Handbook of Ternary Alloy Phase Diagrams, Vols. 1-10, ASM International, Materials Park, OH (1995).

13. M. L. Cohen. Solid State Comm. 107, 589 (1998).

14. L. Gao, Y. Y. Xue, F. Chen, Q. Xiong, R. L. Meng, D. Ramirez, C. W. Chu, J. H. Eggert, H. K. Mao. Phys. Rev. B50 4260-4263 (1994).

15. Structures of oxides and halides can be found in the Inorganic Crystal Structure Database published by FIZ Karlsruhe and the Gmelin Institute.

16. M. Krack and M. Parrinello. Phys. Chem. Chem. Phys. 2, 2105 (2000); G. Lippert, J. Hutter, M. Parrinello. Theor. Chem. Acc. 103, 124 (1999). 
17. E. Reddington, A. Sapienza, B. Gurau, R. Viswantathan, S. Sarangapani, E. S. Smotkin, T. Mallouk. Science 280, 1735 (1998).

18. B. Knigge, A. Hoffmann, D. Lederman, D. C. Vier, S. Schultz, I. K. Schuller. J. Appl. Phys. 81, 2291 (1997). 